\title{
Continent-Scale Sampling Reveals Fine-Scale Turnover in a Beneficial Bug Symbiont
}

OPEN ACCESS

Edited by:

Martin Kaltenpoth,

Johannes Gutenberg University

Mainz, Germany

Reviewed by:

Yoshitomo Kikuchi,

National Institute of Advanced Industrial Science and Technology

(AIST), Japan

Laura V. Flórez,

Johannes Gutenberg University

Mainz, Germany

Julia Ferrari,

University of York, United Kingdom

${ }^{*}$ Correspondence:

Alison Ravenscraft

alison.ravenscraft@uta.edu

${ }^{\dagger}$ Present address:

Alison Ravenscraft,

Department of Biology, The University of Texas at Arlington, Arlington, TX,

United States

Margaret W. Thairu,

Department of Bacteriology, University of Wisconsin-Madison,

Madison, WI, United States

Specialty section:

This article was submitted to Microbial Symbioses,

a section of the journal

Frontiers in Microbiology

Received: 23 January 2020

Accepted: 19 May 2020

Published: 19 June 2020

Citation:

Ravenscraft A, Thairu MW, Hansen AK and Hunter MS (2020) Continent-Scale Sampling Reveals Fine-Scale Turnover in a Beneficial Bug Symbiont.

Front. Microbiol. 11:1276. doi: 10.3389/fmicb.2020.01276

\author{
Alison Ravenscraft ${ }^{1 *}$, Margaret W. Thairu' ${ }^{2 t}$, Allison K. Hansen ${ }^{2}$ and Martha S. Hunter ${ }^{3}$ \\ ${ }^{1}$ Center for Insect Science, University of Arizona, Tucson, AZ, United States, ${ }^{2}$ Department of Entomology, University \\ of California, Riverside, Riverside, CA, United States, ${ }^{3}$ Department of Entomology, University of Arizona, Tucson, AZ, \\ United States
}

Many members of animal-associated microbial communities, including the gut flora, are acquired from their host's environment. While many of these communities are species rich, some true bugs (Hemiptera) in the superfamilies Lygaeoidea and Coreidae allow only ingested Burkholderia to colonize and reproduce in a large portion of the midgut. We studied the spatial structuring of Burkholderia associated with a widespread omnivorous bug genus, Jalysus (Berytidae). We sampled Wickham's stilt bug, Jalysus wickhami, across the United States and performed limited sampling of its sister species, the spined stilt bug Jalysus spinosus. We asked: (1) What Burkholderia strains are hosted by Jalysus at different locations? (2) Does host insect species, host plant species, or location influence the strain these insects acquire? (3) How does Burkholderia affect the development and reproductive fitness of J. wickhami? We found: (1) Sixty-one Burkholderia strains were present across a sample of 352 individuals, but one strain dominated, accounting for almost half of all symbiont reads. Most strains were closely related to other hemipteran Burkholderia symbionts. (2) Many individuals hosted more than one strain of Burkholderia. (3) J. wickhami and J. spinosus did not differ in the strains they hosted. (4) Insects that fed on different plant species tended to host different Burkholderia, but this accounted for only $4 \%$ of the variation in strains hosted. In contrast, the location at which an insect was collected explained $27 \%$ of the variation in symbiont strains. (5) Burkholderia confers important fitness benefits to J. wickhami. In laboratory experiments, aposymbiotic (Burkholderia-free) insects developed more slowly and laid fewer eggs than symbiotic (Burkholderia-colonized) insects. (6) In the lab, nymphs sometimes acquired Burkholderia via indirect exposure to adults, indicating that horizontal symbiont transmission can occur via adult insect-mediated enrichment of Burkholderia in the local environment - a phenomenon not previously reported in bug-Burkholderia relationships. Taken together, the results suggest that for these bugs, critical nutritional requirements are outsourced to a highly diverse and spatially structured collection of Burkholderia strains acquired from the environment and, occasionally, from conspecific adults.

Keywords: symbiosis, spatial structure, Burkholderia, Hemiptera, Jalysus, environmental acquisition 


\section{INTRODUCTION}

Symbioses between eukaryotes and microbes are ancient, diverse, ubiquitous, and critical to the biology of many multicellular organisms (McFall-Ngai et al., 2013; Martin et al., 2017). Examples of these symbioses are widespread within a diversity of terrestrial insects (Buchner, 1965; Douglas, 2011, 2015). Across the insect symbiosis literature, transmission mode-the way in which a host insect acquires its symbiotic microbial partnerhas been emphasized as one of the major factors that determines the ecological and evolutionary costs and benefits incurred by partners in these relationships (Ebert, 2013; Salem et al., 2015). It is not, however, generally a predictor of microbial virulence (Ebert, 2013). A host insect may acquire its microbial partner vertically from its parents, horizontally from other conspecifics, or environmentally - directly from its surroundings. These different acquisition modes result in different ecological and evolutionary consequences: the evolutionary interests of a strictly vertically transmitted symbiont are likely to be closely aligned with those of its host, while a free-living microbe acquired from the environment may be pathogenic, commensal or mutualistic, depending on the microbial lineage and the host environment (Moran et al., 2008; Ebert, 2013; Salem et al., 2015).

In insects, the best-studied symbioses are stable associations maintained by vertical transmission of the symbiont from mother to offspring. However, acquisition of free-living symbionts from the environment presents a different set of potential challenges and rewards for a host (Salem et al., 2015). An insect dependent on an environmentally acquired symbiont must be able to find the microbe when the host is at the right life stage and, once the microbe is acquired, it must produce the desired benefit with few associated costs or pathogenic effects. However, the insect population might benefit from the increased flexibility of associating with many different symbiont strains that could confer different functions (Douglas, 1998). Furthermore, freeliving microbes directly interact with, and adapt to, their local environment, and environmental symbiont acquisition permits a high degree of lability in partner associations. Acquisition of the right strain can therefore supply the host with instant adaptation to local conditions, and could even promote niche expansion to new diets or climates (Janson et al., 2008; White, 2011; Kikuchi et al., 2012a).

The hemipteran bug-Burkholderia relationship is an emerging model for the study of pairwise, environmentally acquired symbiosis (Takeshita and Kikuchi, 2017; Kaltenpoth and Flórez, 2020). Many hemipteran species in the superfamilies Lygaeoidea and Coreoidea host Burkholderia in sac-like outgrowths called "crypts" in the last region of the midgut, the M4 region (Kikuchi et al., 2011). Typically, after Burkholderia is ingested and colonizes the M4 region in the second instar nymph stage, the passage to the M4 seals and the M4 becomes a symbiotic organ rather than a flow-through gut (Ohbayashi et al., 2015; Kikuchi et al., 2020). Burkholderia cells are continuously pumped back into the anterior section of the gut, the M4B, where they are digested by the host as a source of nutrients (Ohbayashi et al., 2019). Burkholderia is a common soil inhabitant, often found in proximity to plant roots (Garcia et al., 2014) and at least the bean bug, Riptortus pedestris, is known to acquire Burkholderia from the soil (Kikuchi et al., 2007).

Strains of Burkholderia isolated from insects often fall into a phylogenetic clade called the stinkbug-associated beneficial and environmental (SBE) group (Kaltenpoth and Flórez, 2020). Because young nymphs acquire Burkholderia from the environment every generation, associations between host and symbiont species are highly labile (Kikuchi et al., 2007, 2011). While we do not know whether benefits vary among bugBurkholderia systems, in the model bean bug $R$. pedestris, Burkholderia in the gut express genes associated with nitrogenous waste recycling and biosynthesis of essential amino acids and B vitamins (Ohbayashi et al., 2019). The specificity of the association with this one lineage of bacteria, the dedication of large and elaborate gut chambers (crypts) to house high densities of generally a single strain, and the high prevalence of infection (close to 100\%; Kikuchi et al., 2011) of the symbiont in natural host populations suggest that Burkholderia provide consequential benefits in all of these associations. Rearing bugs without the symbiont has been shown to cause increased mortality and decreased size in Riptortus and Alydus (Alydidae) (Kikuchi et al., 2007; Garcia et al., 2014).

Given the high degree of lability in these relationships and their environmentally acquired nature, the factors that determine which strain a nymph will acquire are still poorly understood. We asked how the Burkholderia strain hosted by bugs in the genus Jalysus (Berytidae) varied with location, host insect species (J. wickhami versus J. spinosus), and host plant species. We characterized the alpha and beta diversity of Jalysus-associated Burkholderia strains across the United States, predicting that Burkholderia strains would be spatially structured as a result of climate or isolation by distance and would be correlated with the insect's host plant species. To our knowledge, this is the largest spatial scale studied to date for a bug-Burkholderia symbiosis. Our results provide a better understanding of how communities of symbiotic Burkholderia are structured.

The Jalysus-Burkholderia symbiosis is unusual and puzzling because Jalysus is an omnivore, requiring both plants and scavenged or live prey for survival and reproduction (Elsey and Stinner, 1971). Many insects that depend on microbial symbionts use them to compensate for an unbalanced diet, such as a low nitrogen plant-based diet, or a B-vitamin-poor diet of blood (Buchner, 1965); however, we expect Jalysus' diet to be balanced. We therefore asked whether Burkholderia does, in fact, provide a benefit to Jalysus as it has been shown to do for herbivorous bugs. We show that Burkholderia is indeed important for normal development and reproductive success in Jalysus.

\section{MATERIALS AND METHODS}

\section{Sample Collection and DNA Extraction}

Insects were collected from 22 sites in California, Arizona, Illinois, North Carolina, or South Carolina between July 2015 and June 2017 (Supplementary Table S1) and preserved in 95\% ethanol. Jalysus often live in aggregations with overlapping generations (Figure 1). Nymphs cannot fly and therefore usually 


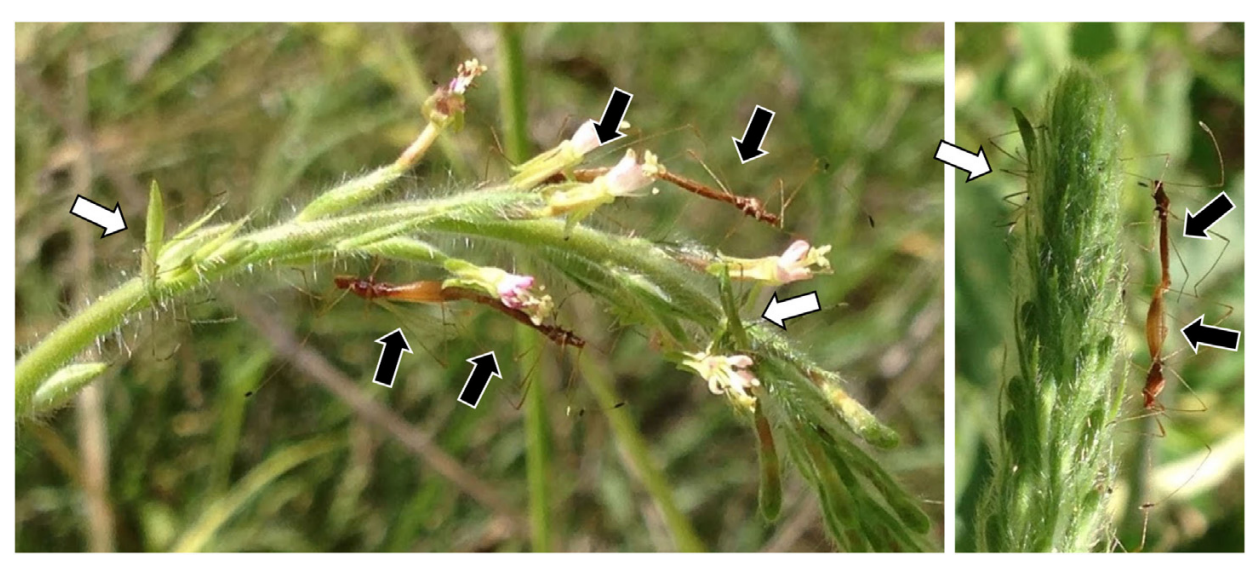

FIGURE 1 | Jalysus in the field. Overlapping generations of Jalysus wickhami often live in close proximity. Here adults (black arrows) and nymphs (white arrows) are feeding on the same flower stalk of Gaura parviflora.

complete development on a single host plant or patch of contiguous plants. Although adults can fly, they tend to be relatively sedentary and often return to the same host plant when disturbed. We captured 293 adults, 121 fifth instar nymphs, 43 fourth instar nymphs, and 2 third instar nymphs, plus 6 individuals for which developmental stage was not noted. This resulted in a total of 465 insects, mostly of Jalysus wickhami (424 individuals) but also including Jalysus spinosus (41 individuals).

DNA was extracted from whole insect bodies with the DNeasy Blood and Tissue Kit (Qiagen, Germantown, MD). While processing our samples, we also performed the protocol for DNA extraction on 10 empty tubes interspersed throughout the samples. These blank extractions were processed simultaneously with and identically to the samples, including all downstream lab work and sequencing, and allowed us to assess both environmental contamination and sample cross-contamination.

\section{Illumina Library Preparation and Sequencing}

We amplified the V3-V4 hypervariable region of the 16S rRNA using the universal bacterial primer set $341 \mathrm{f}\left(5^{\prime}\right.$-CCTACGGGNGGCWGCAG-3') and 785r (5'GACTACHVGGGTATCTAATCC-3') (Klindworth et al., 2013). Primers included an overhang adapter to enable downstream addition of indexes for sample differentiation, as recommended by Illumina (Illumina Inc, 2013). Samples were amplified in a volume of $25 \mu \mathrm{L}$ with the following reaction concentrations: $0.2 \mu \mathrm{M}$ forward primer, $0.2 \mu \mathrm{M}$ reverse primer, $0.2 \mathrm{mM}$ dNTPs, 0.65U OneTaq Hot Start polymerase (New England Biolabs), 1X OneTaq standard reaction buffer (New England Biolabs), and $2 \mathrm{uL}$ DNA extract. Thermocycler settings were: Denaturation at $95^{\circ} \mathrm{C}$ for $3 \mathrm{~min}$ followed by 30 cycles of denaturation at $95^{\circ} \mathrm{C}$ for $30 \mathrm{~s}$, primer annealing at $55^{\circ} \mathrm{C}$ for $30 \mathrm{~s}$, and extension at $68^{\circ} \mathrm{C}$ for $50 \mathrm{~s}$, with a final extension of $68^{\circ} \mathrm{C}$ for $10 \mathrm{~min}$. PCR products were visualized with gel electrophoresis and cleaned with magnetic beads (Rohland and Reich, 2012). In addition to the extraction blanks, every 96-well plate of samples included at least one blank sample of PCR water. All blanks were included in all downstream lab work and sequencing.

In a second short amplification, each sample was indexed with a unique pair of 8-nt barcodes attached to the forward and reverse strands (Hamady et al., 2008). We amplified $2.5 \mathrm{uL}$ of each cleaned PCR product in a volume of $25 \mu \mathrm{L}$ with $0.2 \mu \mathrm{M}$ of a barcoded forward primer, $0.2 \mu \mathrm{M}$ of a barcoded reverse primer, $0.2 \mathrm{mM}$ dNTPs, 0.65U OneTaq Hot Start polymerase, and 1X OneTaq standard reaction buffer. The thermocycler program was the same as that of the initial amplification, with two modifications: the annealing temperature was $50^{\circ} \mathrm{C}$ and 8 amplification cycles were performed. PCR products were again visualized with gel electrophoresis and cleaned with magnetic beads.

The cleaned, dual-indexed 16S rRNA amplicons were quantified fluorometrically (Qubit DS DNA HS assay, Qiagen) and an equal mass of each sample was bidirectionally sequenced on an Illumina Mi-Seq platform using $2 \times 300$ chemistry, with samples split between two runs: one at the University of Arizona Genetics Core and one at the Arizona State Genomics and Bioinformatics Core.

\section{Sequence Data Processing}

We used the program cutadapt (Martin, 2011) to remove priming sites and poor quality bases at the $5^{\prime}$ and $3^{\prime}$ ends of the sequences. The resulting sequences were processed in R using the "DADA2" package (Callahan et al., 2016) as follows: Using the filterAndTrim function, reads were truncated at the first instance of a quality score less than or equal to 2, and reads that contained any unassigned bases (Ns) or had an overall expected error score higher than 2 were discarded. We used the package's eponymous DADA2 algorithm which employs the run-specific error rate, quality scores, and the number of times each sequence was observed to infer the true biological sequences that were present, allowing analysis at the level of bacterial strains (Callahan et al., 2016). Analysis of sequence variants, rather than use of older clustering methods that generate taxonomic units at a 97 or $99 \%$ similarity cutoff, is the current recommended best practice for 
amplicon data (Knight et al., 2018). Forward and reverse reads were merged and sequences less than 401 base pairs in length were discarded, resulting in a median sequence length of 427 base pairs. De novo chimera checking and removal were performed with the removeBimeraDenovo function. Taxonomy was assigned using the RDP classifier with the SILVA nr v123 database as the training set (Wang et al., 2007; Quast et al., 2013).

Eleven Burkholderia sequence variants were detected in the extraction and PCR blanks (Supplementary Figure S1). These included some of the most common sequence variants in the dataset, including SV1. The most common bacteria present in a library of samples are almost always detected in Illumina blanks due to phenomena such as index-swapping (Costello et al., 2018) and low-levels of sample cross-contamination. However, the sequence variants varied markedly across blanks: ten out of eleven were only detected in a single blank. SV1, the most common sequence variant in the dataset, was detected in 5 out of 15 blanks. To identify true contaminants, we compared the prevalence of each sequence variant in insect samples versus blanks using the R package "decontam" with default parameters (Davis et al., 2018). Eighteen sequence variants, including one Burkholderia (SV156), were classified as contaminants. These were removed from the dataset.

\section{Molecular Phylogenetic Analysis}

We constructed a phylogeny of all Burkholderia sequence variants that were present in the dataset after rarefaction to 800 reads along with selected representatives of different Burkholderia from NCBI's GenBank (taken from Kuechler et al., 2016). Accession numbers are available in Supplementary Table S2. Sequences of the 16S rRNA gene were aligned using MAFFT (Katoh and Standley, 2013) with the default settings. Maximum-likelihood phylogenetic trees were inferred with the GTR+Gamma model of nucleotide substitution using RAxML version 8.2.10 (Stamatakis, 2014) on the CIPRES Science Gateway (Miller et al., 2010). Node support values were calculated using rapid bootstrapping, which was halted automatically based on the MRE criterion.

\section{Insect Rearing Experiments}

Jalysus wickhami proved difficult to maintain in an experimental setting and mortality of young nymphs was high (Supplementary Table S3). (However, mortality did not differ between the symbiotic and aposymbiotic treatments; Fisher's exact test, odds ratio $=1.26, p=0.6$ ). We therefore performed three iterations of an experiment to compare the development time and/or reproductive output of $J$. wickhami reared with and without symbiotic Burkholderia. Rearing conditions are summarized in Table 1. In the first two experiments, a single first-instar nymph freshly hatched from an egg in an aseptic container - was placed in a clean plastic box that contained a tomato leaflet in water and a source of animal protein (Supplementary Figure S2).

In the first iteration, dead Drosophila flies were provided as the protein source. From the first through the third instars, each nymph assigned to the "symbiont positive" treatment was reared in the same box as a single adult male which was normally infected with Burkholderia. These adult males were collected from our laboratory colony of Jalysus wickhami, in which insects freely acquired Burkholderia from the potting soil in which the host plant (tomato) was grown. Each male was maintained on a single tomato leaf - which had not previously been exposed to Jalysus - in the plastic rearing box for several days prior to introduction of the experimental nymph. Males that died were replaced with another male from the lab colony. In contrast, nymphs assigned to the aposymbiotic ("symbiont negative") treatment were kept on leaves which were not exposed to any adult insects. This experiment was performed in an incubator kept at $28^{\circ} \mathrm{C}$ with a $16: 8$ hour day:night cycle and $65 \%$ relative humidity. Upon reaching adulthood, insects were immediately preserved in $95 \%$ ethanol for later DNA extraction to determine Burkholderia infection status.

In the second iteration of the experiment, an artificial diet of homogenized calf liver, fatty beef, and sugar water was provided as the protein source (Cohen, 1985). From the first through the third instars, a suspension of live Burkholderia cells was mixed into the diet of nymphs in the symbiont positive treatment; an equal volume of distilled water was mixed into the diet of the aposymbiotic nymphs. Symbiotic insects were infected with Burkholderia strain TF1N1, which we isolated from insects collected on tobacco in North Carolina. This strain was 100\% identical in sequence (across 408 bp of the V3-V4 region of the 16 s rRNA) to SV1 in our Illumina amplicon data. The rearing experiment was performed in the laboratory at room temperature $\left(\sim 22^{\circ} \mathrm{C}\right)$ under ambient light and humidity conditions. Upon reaching adulthood, males were preserved in 95\% ethanol for later DNA extraction. Females were maintained for an additional 2 weeks to collect data on reproductive output. A normally infected male from the lab colony was added to the cage of each female and the insects were allowed to mate freely. Eggs were collected and counted every other day. After 2 weeks, the females were preserved in $95 \%$ ethanol.

Since only six females (out of an initial 48 nymphs) successfully completed the reproductive output measurement in the second iteration, we performed a third iteration to obtain more data on reproductive fitness. To provide a source of the symbiont, six adult J. wickhami were maintained on a tomato leaf in a plastic box for a minimum of 5 days; these adults (and any eggs they had laid) were then removed from the box and eight fresh, experimental eggs were placed in the box. For the aposymbiotic treatment, leaf sprigs were kept in a box without adults for a minimum of 5 days prior to egg introduction. Nymphs in both treatments were switched to a new box (that was either exposed or un-exposed to six adults, as appropriate) every three to four days until the end of the third instar. At the beginning of the fifth instar, each nymph was transferred to a fresh box of its own. Upon reaching adulthood, a male was introduced to each female's cage and the insects were allowed to mate freely. Eggs were collected and counted every other day. After 2 weeks, the females were preserved in 95\% ethanol.

DNA of the preserved adults was extracted using the DNeasy Blood and Tissue Kit (Qiagen, Germantown, MD, United States) and diagnostic PCR was performed using the Burk16sF/Burk16sR primer set $\left(5^{\prime}\right.$ TTTTGGACAATGGGGGCAAC-3', 5'-GCTCTTGCGTAGC AACTAAG-3'; Kikuchi et al., 2005) to verify Burkholderia 
TABLE 1 | Experimental rearing conditions.

\begin{tabular}{|c|c|c|c|}
\hline Condition & Iteration 1 & Iteration 2 & Iteration 3 \\
\hline Temperature & $28^{\circ} \mathrm{C}$ & Ambient $\left(\sim 22^{\circ} \mathrm{C}\right)$ & $28^{\circ} \mathrm{C}$ \\
\hline Light:Dark (hours) & $16: 8$ & Ambient & $16: 8$ \\
\hline Relative Humidity & $65 \%$ & Ambient & $65 \%$ \\
\hline Protein source & Dead Drosophila & Artificial diet (beef) & Dead Drosophila \\
\hline Burkholderia source & Single adult male (instars 1-3) & Live cells mixed into diet (instars 1-3) & Leaves exposed to six adults (instars 1-3) \\
\hline Direct contact with adults? & Yes & No & No \\
\hline Life stage at start & 1st instar & 1st instar & egg \\
\hline Nymphs reared per box & 1 & 1 & Up to 8 until fifth instar, then 1 \\
\hline
\end{tabular}

infection status. Each $10 \mathrm{uL}$ PCR reaction contained $0.4 \mu \mathrm{M}$ forward primer, $0.4 \mu \mathrm{M}$ reverse primer, $1.6 \mathrm{mM}$ dNTPs, 0.75U Taq (New England Biolabs), 1X ThermoPol buffer (NEB M0267), and $1 \mathrm{uL}$ DNA extract. The thermocycler program was: Denaturation at $95^{\circ} \mathrm{C}$ for $4 \mathrm{~min}$, followed by 40 cycles of denaturation at $94^{\circ} \mathrm{C}$ for $30 \mathrm{~s}$, primer annealing at $55^{\circ} \mathrm{C}$ for $1 \mathrm{~min}$, and extension at $68^{\circ} \mathrm{C}$ for $2 \mathrm{~min}$, with a final extension of $68^{\circ} \mathrm{C}$ for $6 \mathrm{~min}$. PCR products were visualized with gel electrophoresis. When a PCR result disagreed with the experimental treatment (i.e., Burkholderia was detected in an insect assigned to the aposymbiotic treatment or Burkholderia was not detected in an insect assigned to the symbiotic treatment), the insect was reassigned to the diagnostic PCR result.

We did not sequence the experimental insects nor the adults that served as Burkholderia sources in Iterations 1 and 3 . However, we did sequence 16 adults from the laboratory colony from which the source adults were derived: Fourteen were monoassociated with SV1 and two were monoassociated with SV17. SV1 is either the same strain as, or a very close relative of, the TF1N1 Burkholderia isolate we used to infect nymphs in Iteration 2, therefore most or all of the insects in our experiments were likely infected with the same Burkholderia strain.

\section{Statistical Analyses}

To explore the dominant bacteria present in Jalysus, we rarefied the full $16 \mathrm{~S}$ dataset to 1000 reads per sample to control for differences among samples in sequencing depth and calculated the total number of reads assigned to each sequence variant. For the rest of our analyses, we subset the raw (unrarefied) 16S data to sequence variants assigned to the genus Burkholderia. In order to generate simple summary statistics (e.g., numbers of strains hosted) and pie charts, we controlled for sample-wise differences in sequencing depth by rarefying all Burkholderia sequences to 800 reads per sample. We chose 800 reads because it struck a balance between retaining a large number of insects in the dataset (352 individuals out of 465) and retaining a high minimum number of reads per sample for a single bacterial genus. We also generated bar plots of strain composition within individual insects using the $\mathrm{R}$ package "phyloseq" (McMurdie and Holmes, 2013).

For our statistical analyses, we took the full set of unrarefied Burkholderia sequences and discarded samples below a minimum threshold of 800 Burkholderia reads. We removed insects from sites with fewer than 8 collected individuals and from host plants of unknown species or from which fewer than 14 individuals were collected. To test for community-level differences in the Burkholderia hosted by wild Jalysus, Bray-Curtis distances between samples were calculated from unrarefied, proportionnormalized data. (Proportion normalization performs best for this type of test; McMurdie and Holmes, 2014.) We used PERMANOVA (the adonis test in the R package "vegan"; Oksanen et al., 2015) to test for dissimilarity between the insect species (wickhami versus spinosus) while accounting for differences between eastern and western regions of the United States. The same method was also used to test for differences in the Burkholderia community of insects on different plant species while accounting for differences between collection sites. Finally, we tested for differential abundance of individual SVs between sample groups using the $\mathrm{R}$ package "DESeq2" adapted for microbiome data (Anders and Huber, 2010; McMurdie and Holmes, 2014).

We used a linear model to assess how development time from hatching to adulthood was correlated with symbiont infection status (as determined by diagnostic PCR), experimental iteration, and the sex of the nymph. We used a generalized linear model with Poisson error distribution to assess how reproductive output (number of eggs laid by a female during the first 2 weeks of adulthood) was correlated with a female's symbiont treatment and experimental iteration. We found the best-fit fixed effects structure for both models using backward model selection with likelihood ratio tests.

\section{RESULTS}

After sequence processing, quality filtering, and removal of contaminant sequence variants, we obtained 3,134,451 bacterial sequences from 465 insects. To verify that the insects' wholebody bacterial communities were dominated by known insectassociated bacteria, we first rarefied the entire 16S dataset to 1000 reads per sample. After rarefaction, a total of 341 bacterial sequence variants (SVs) were detected. Many of the most abundant SVs were Burkholderia, but other common genera included Wolbachia, Bartonella, and Commensalibacter; these genera are commonly found in association with insects (Supplementary Table S4).

Next, we subset the data to the genus Burkholderia. After applying a minimum cutoff of 800 Burkholderia reads per insect, 
we retained 322 individuals of $J$. wickhami from 20 sites and 30 individuals of $J$. spinosus from 2 sites. We detected a total of 61 Burkholderia sequence variants across these individuals.

\section{Phylogenetic Placement of Burkholderia Associated With Jalysus spp.}

Because Illumina amplicons are relatively short ( $\sim 27$ bp) our phylogeny did not have strong support (Figure 2). However, the best-fit phylogeny (Supplementary Figure S3) recovered the same Burkholderia groups previously described by Kuechler et al. (2016) - specifically, the " $B$. cepacia complex and B. pseudomallei" (BCC\&P) group; the "plantassociated beneficial and environmental" (PBE) group which contains the "insect-associated and plant-associated beneficial and environmental" (iPBE) group; and the "stinkbug-associated beneficial and environmental" (SBE) group which contains subgroups associated with the Coreidae and Stenocephalidae. However, we did not recover the same basal relationships between groups found by Kuechler et al. (2016).

According to our best-fit phylogeny, our Burkholderia SVs derived from all major Burkholderia clades except for the BCC\&P, but the 17 most common sequence variants (those accounting for greater than $0.1 \%$ of the reads in at least 5 insects) were all members of the SBE clade (Supplementary Figure S3). The four most abundant sequence variants - SV1, SV6, SV8, and SV21 appeared to be most closely related to symbionts of insects in the family Coreidae (Supplementary Figure S3).

\section{Alpha and Beta Diversity of Burkholderia Variants Associated With Jalysus}

After rarefaction to 800 Burkholderia reads per sample, individual insects hosted a median of 1 (interquartile range (IQR) 12 , $\max =9$ ) sequence variants. Sequence variant abundances were uneven within hosts, with one variant being substantially more abundant than the other(s) in each insect (Figure 3). Unexpectedly, one sequence variant, SV1, was widespread across the continental United States; it accounted for $48 \%$ of all Burkholderia reads. After removing sites with fewer than 8 individuals, SV1 was the most abundant sequence variant in most (13 out of 17 ) sites. There were only two sites in which SV1 was not detected.

The composition of symbiont communities differed between the eastern and western United States (PERMANOVA: $d f=1$, $F=16.4, p<0.001$ ), though the overall amount of variation explained was small $\left(R^{2}=0.05\right)$. Seven sequence variants displayed an east-west divide in their distributions: SVs 6, 14, 21 and 24 were detected predominantly on the western side of the United States and SVs 8, 18, and 22 were largely limited to the east (DESeq2, all $p<0.001$; Figure 4). After accounting for differences between the east and west, symbiont communities did not differ between J. spinosus and J. wickhami (PERMANOVA: $d f=1, F=0.7, p=0.6$ ).

Differences between sites accounted for a high percentage of variation in Burkholderia communities (PERMANOVA: $\left.d f=14, F=7.4, p<0.001, R^{2}=0.27\right)$. On average, Jalysus within the same site were more likely to host the same sequence variant than Jalysus from different sites (average BrayCurtis within populations $=0.53$; average Bray-Curtis between populations $=0.74 ; t=3.8, d f=27, p<0.001$. For reference, a Bray-Curtis dissimilarity of 1 indicates that no SVs were shared; a Bray-Curtis dissimilarity of 0 indicates that all SVs were shared at identical relative abundances).

After accounting for site differences, host plant species were also found to differ in symbiont community composition, though the variation explained was small (PERMANOVA: $d f=2, F=7.2$, $\left.p<0.001, R^{2}=0.04\right)$. Insects collected from the same host plant species were more likely to host the same sequence variant than insects collected from different host plant species (average BrayCurtis within plant species $=0.65$; average Bray-Curtis between plant species $=0.80 ; t=2.3, d f=15, p=0.03)$. At two sites, we were able to collect insects from different host plant species in close proximity. At site Sac3, J. wickhami were collected from a small cluster of contiguous Zauschneria californica plants and from an individual Oenothera plant less than 5 meters away. Burkholderia strain SV21 was significantly associated with Oenothera at this site (DESeq2, $p<0.001$; Figure 3). At site Dav4, we collected J. wickhami from a large patch of about 30 contiguous Gaura lindheimeri and from a small patch of about 5 contiguous Zauschneria californica plants roughly 200 meters away. No Burkholderia strains differed in abundance between the two host plant species at this site (DESeq2, all $p>0.7$; Figure 3).

\section{Burkholderia Colonization Rates}

We performed diagnostic PCR to check the Burkholderia colonization status of all insects that reached adulthood across the three iterations of the rearing experiment. In the first iteration, only four out of 14 individuals (29\%) acquired Burkholderia in the symbiont treatment (Table 2). However, in the second and third iterations, all but one individual successfully acquired Burkholderia when exposed to a source of the symbiont (Table 2). Almost all insects in the aposymbiotic treatments were confirmed to be Burkholderia-free, however, two individuals in Iteration 3 did acquire Burkholderia despite lack of intentional exposure to a symbiont source (Table 2 ). In total, only $2 / 26$ (8\%) of nymphs that were not intentionally exposed to Burkholderia acquired the symbiont, while 20/32 (63\%) of nymphs exposed either indirectly via adults, or directly via cultured cells acquired the symbiont $\left(X^{2}=16, d f=1, p<0.001\right)$. For the remainder of our analyses, symbiont colonization status was assigned based on an insect's diagnostic PCR result rather than its original experimental treatment.

\section{Effects of Burkholderia on Development and Fitness of Jalysus}

We compared development time and reproductive output of J. wickhami reared with or without the Burkholderia symbiont. Due to differences in diet and rearing temperature, insects in the first iteration of this experiment developed 12.1 days faster than insects in the second iteration $(d f=1, t=6.15$, $p<0.001)$. After accounting for these differences by including the experimental iteration as a factor in the model, we found aposymbiotic nymphs took 8.8 days longer to reach adulthood 


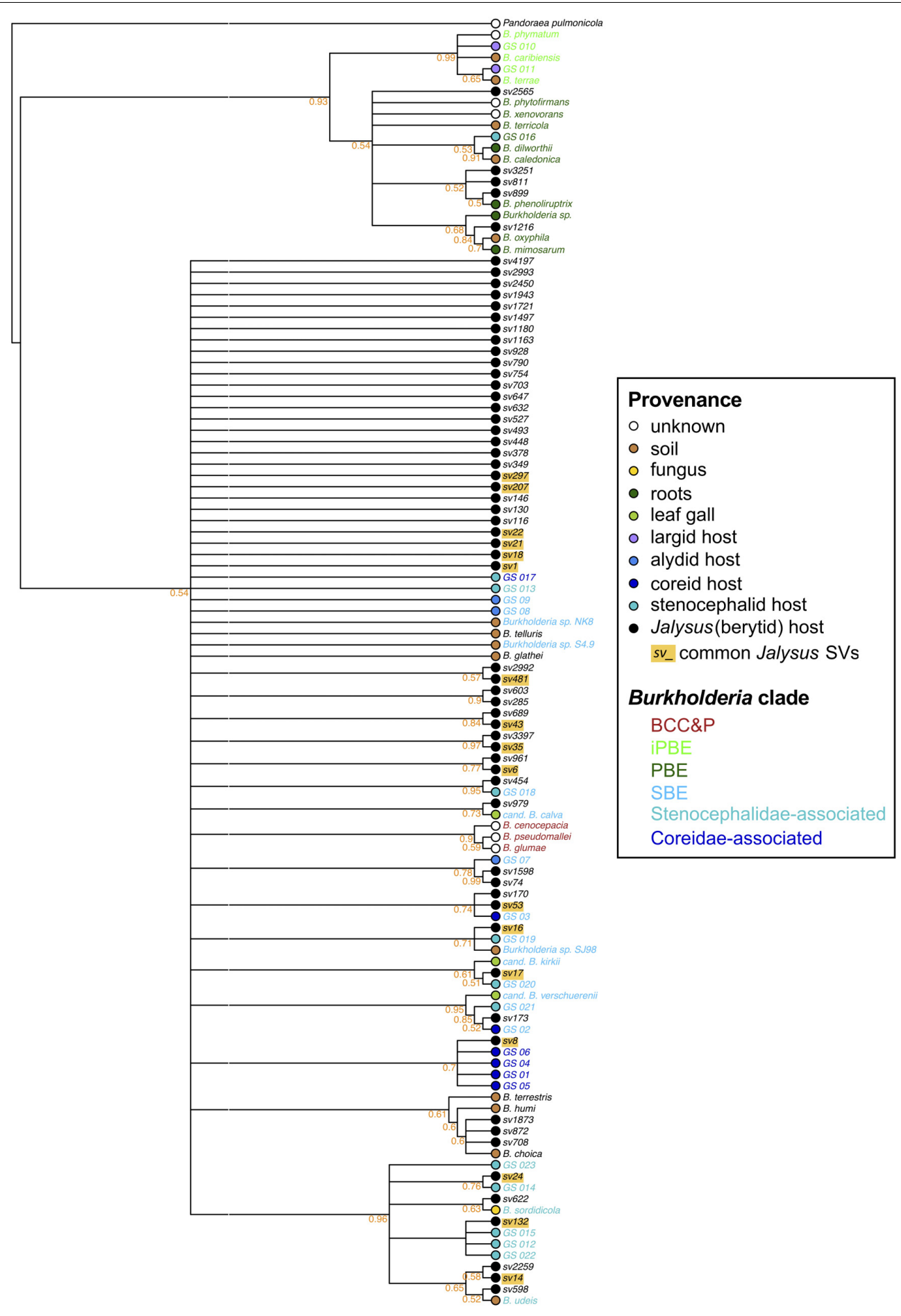

FIGURE 2 | Phylogenetic placement of Burkholderia associated with Jalysus spp. Consensus maximum-likelihood phylogeny of the Burkholderia sequence variants detected in this study (names starting "sv" and labeled with a black dot) plus the 16S rRNA sequences of selected strains downloaded from GenBank. Where known, the source of each Burkholderia variant is indicated by colored dots. Text labels of GenBank strains are colored according to their membership in several named groups as proposed by Kuechler et al. (2016): SBE = stinkbug-associated beneficial and environmental; PBE = plant-associated beneficial and environmental; iPBE = the insect-associated subclade of the PBE group; BCC\&P = B. cepacia complex and B. pseudomallei group; a clade associated with coreid bugs; and a clade associated with stenocephalid bugs. The most common sequence variants in Jalysus (those accounting for greater than $0.1 \%$ of the reads in at least five insects) are highlighted in yellow. Sequence variants from this study were 427 or 428 base pairs in length. GenBank sequences varied from 1231 to 1731 bp in length. The tree was rooted with the outgroup Pandoraea pulmonicola. Node support values were calculated using rapid bootstrapping which was halted automatically based on the MRE criterion. Nodes with less than $50 \%$ support have been collapsed. The corresponding best-fit maximum-likelihood tree is provided in Supplementary Figure S3. 


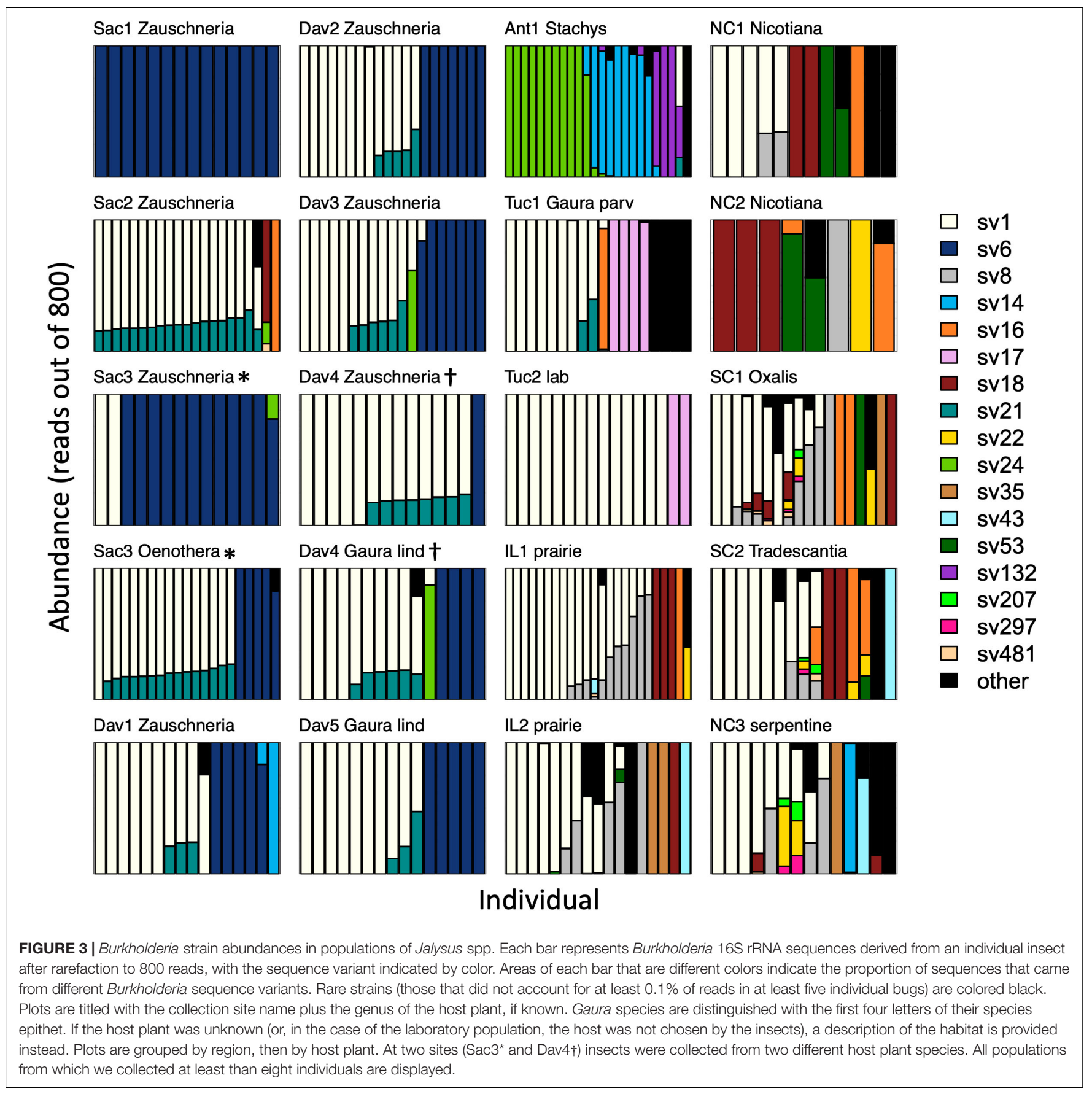

than symbiotic nymphs on average ( $d f=1, t=4.4, p<0.001$; Figure 5A and Supplementary Figure S4A), representing a $34 \%$ increase in the duration of development compared to symbiotic nymphs.

Also due to differences in diet and rearing temperature, insects in the second iteration of this experiment laid 0.9 fewer eggs ( $d f=1, z=5.1, p<0.001)$ than insects in the third iteration. After accounting for these differences, aposymbiotic females laid 2.1 fewer eggs in their first 2 weeks of adulthood than symbiotic females ( $d f=1, z=5.4, p<0.001$; Figure 5B and Supplementary Figure S4B). We also observed that aposymbiotic adults were paler and their exoskeletons appeared to be weaker and more pliable than those of symbiotic adults (Supplementary Figure S5). Pale coloration of aposymbiotic individuals has been reported in several stinkbug species (Hosokawa et al., 2006, 2013; Kikuchi et al., 2012b, 2016).

When we assigned symbiont colonization status based on the original experimental treatment rather than PCR, results for both development time and reproductive output were qualitatively identical, though differences between symbiotic and aposymbiotic insects were less pronounced (Supplementary Table S5). 


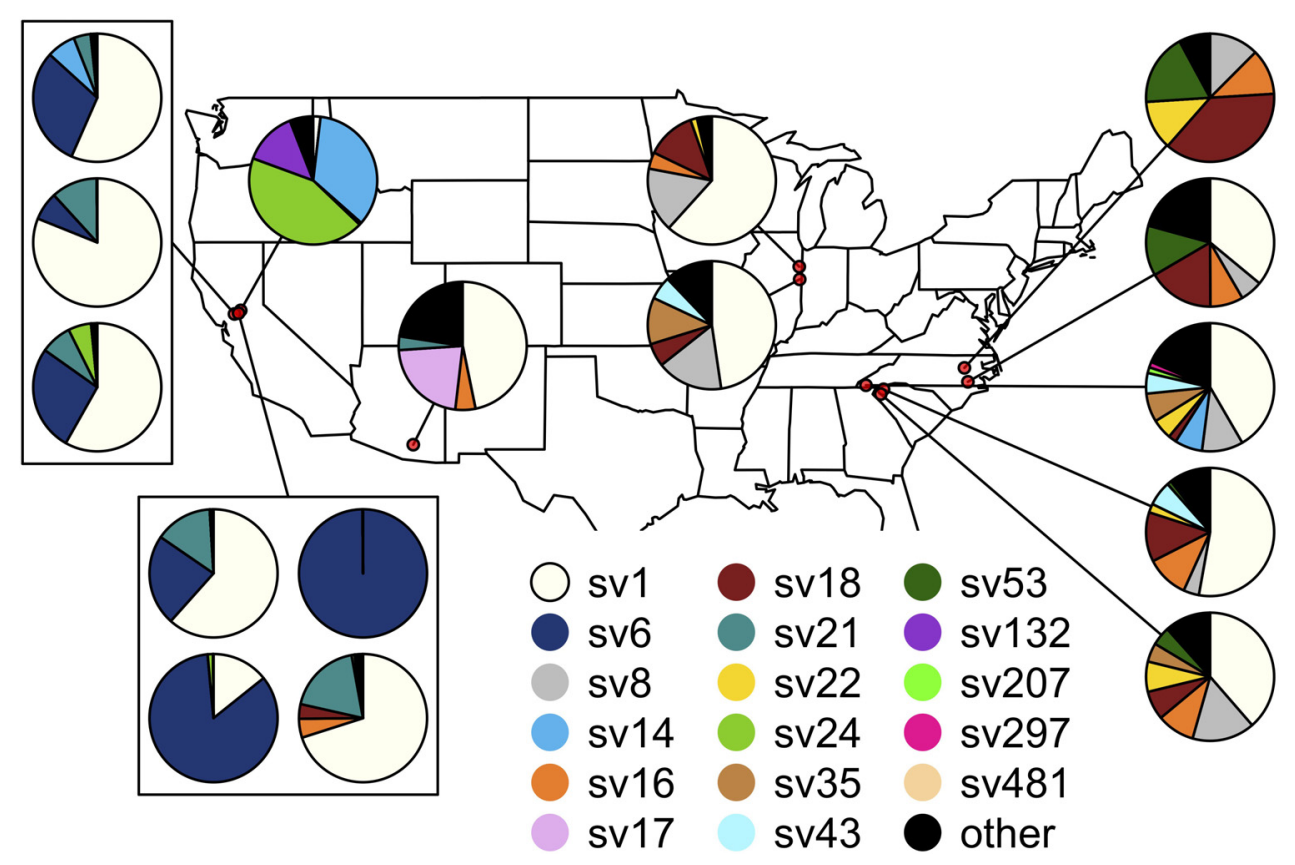

FIGURE 4 | Spatial distribution of the Burkholderia sequence variants hosted by Jalysus. Each pie chart indicates population-level abundances of variants hosted by a pool of 12-24 individual bugs sampled at a single location. Rare strains (those that did not account for at least $0.1 \%$ of reads in at least five individual bugs) are colored black.

\section{DISCUSSION}

We characterized symbiotic Burkholderia associated with a widespread, omnivorous stilt bug genus (Jalysus: Berytidae) across the United States and quantified the net fitness effects of the most common symbiont strain on J. wickhami. Despite high variation in the identity of the strains with which Jalysus associates, Burkholderia appears critical to the insect's development and reproductive success. Without their symbiont, J. wickhami grow slower and lay fewer eggs. Aposymbiotic adults were generally paler and slower moving, suggesting potential deficits in normal melanization. Evidence that J. wickhami depends on a symbiont that is putatively nutritional is especially intriguing given that these stilt bugs are omnivorous and we provided the insect with unlimited protein in our experiments. In this respect, this system is very different from most nutritional symbioses that serve to complement an indigestible, unbalanced or nitrogen poor diet, for example of plant sap, wood, or blood (Buchner, 1965). Even though J. wickhami's diet is nitrogen rich, the insect still depends on Burkholderia. Given the evidence that Burkholderia in gut crypts can recycle nitrogenous wastes such as allantoin and urea, as well as produce essential amino acids and B vitamins (Ohbayashi et al., 2019) stilt bugs might assimilate nutrients more efficiently with their Burkholderia symbiont, even in the presence of ample protein. Perhaps, as in a weevil-Nardonella symbiosis, the symbiont boosts the supply of a particular nutrient beyond levels that feeding or host synthesis could provide during critical developmental timepoints (Anbutsu et al., 2017). It is also possible that Burkholderia synthesizes a non-nitrogenous nutrient for Jalysus, and recent results suggest that hosts may receive many different nutrients simultaneously via digestion of Burkholderia cells in the midgut region directly anterior to the M4, the M4B (Ohbayashi et al., 2019). Alternatively, it's possible that the long association between stilt bugs and Burkholderia has resulted in relaxed selection on the host insect's genome for synthesis of a critical nutrient that Burkholderia provides, regardless of the abundance of the nutritional building blocks in the bug diet.

As has been found in other bug-Burkholderia symbioses, the relationship between Jalysus and Burkholderia appeared highly promiscuous. Jalysus associated with over 60 symbiont strains and these did not differ consistently between the sister insect species (wickhami and spinosus). Although one strain, SV1, was dominant and geographically widespread across insect populations, others were more localized: seven sequence variants were associated with either eastern or western sites. However, differences between the east and west explained a small amount $(5 \%)$ of the total variation in symbiont communities. Insects that fed on different host plant species did tend to host different Burkholderia, but this also only accounted for a small amount (4\%) of the variation in hosted symbiont communities. Furthermore, at two sites where we sampled multiple plant species, only one or zero strains, respectively, differed in abundance between the plant species. In contrast, the specific location at which an insect was collected explained a large amount (27\%) of the variation in symbiont strains. Together, these results suggest that Burkholderia does not play an important role in these insects' host plant specificity, 
TABLE 2 | Sample sizes of nymphs that reached adulthood grouped by assigned Burkholderia treatment and diagnostic PCR results.

\begin{tabular}{|c|c|c|c|c|c|}
\hline Treatment & PCR Result & Iteration 1 & Iteration 2 & Iteration 3 & Total \\
\hline Sym & Sym & 4 & 11 & 5 & 20 \\
\hline Sym & Apo & 10 & 1 & 1 & 12 \\
\hline Apo & Apo & 14 & 8 & 2 & 24 \\
\hline \multirow[t]{2}{*}{ Apo } & Sym & 0 & 0 & 2 & 2 \\
\hline & Total & 28 & 20 & 10 & 58 \\
\hline
\end{tabular}

Apo, aposymbiotic; Sym, symbiotic. Rows highlighted in blue indicate nymphs for which the diagnostic PCR result matched the assigned treatment group.
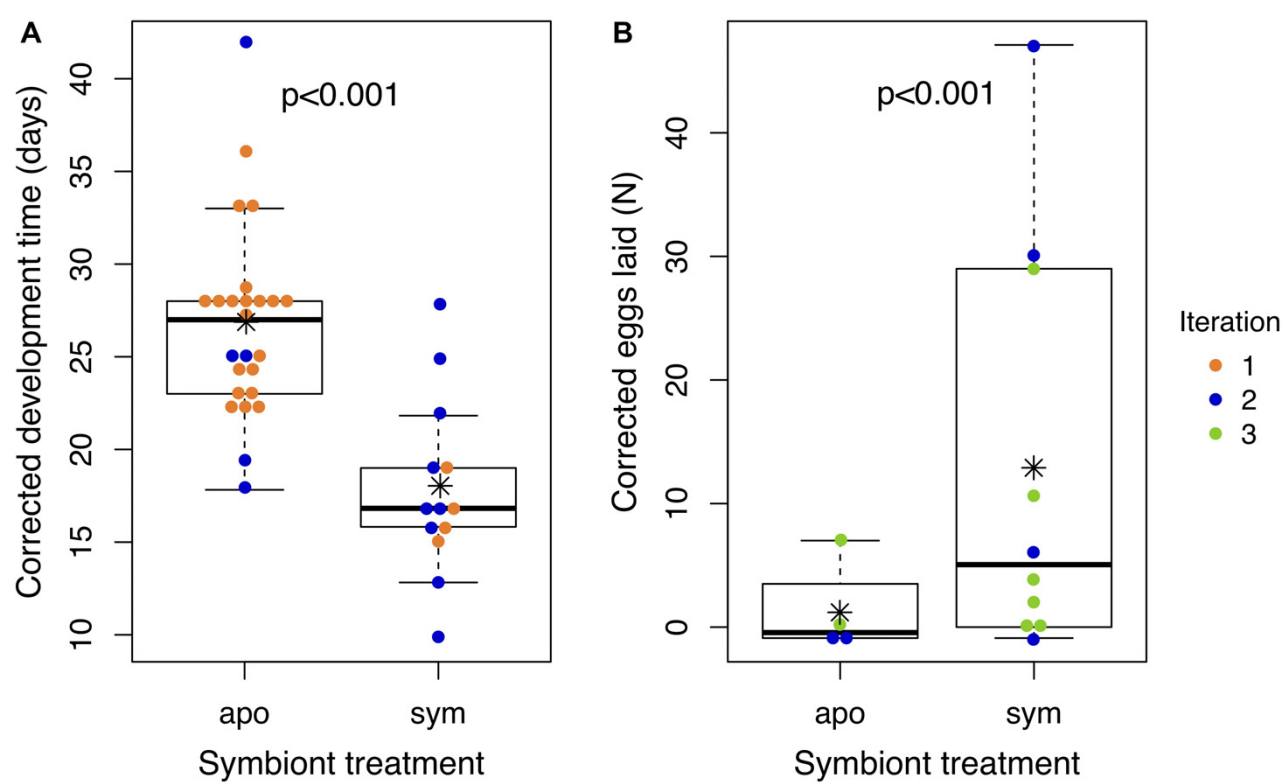

FIGURE 5 | Aposymbiotic insects develop slower and lay fewer eggs than symbiotic insects. Points represent (A) the time an individual nymph took to develop from hatching to adulthood or (B) the number of eggs laid by an individual female over the first 2 weeks of adulthood. All values have been corrected for experimental iteration: development times represent model estimates if all nymphs had been reared in Iteration 1; egg numbers represent model estimates if all females had been reared in Iteration 3. Asterisks denote group means. Box plots depict medians and interquartile ranges of the data. Whiskers are placed at 1.5 times the interquartile range or, if all data fall within this range, they are placed at most extreme value measured. Corresponding raw data are displayed in Supplementary Figure S5.

but local factors drive which strains Jalysus acquires at a fine spatial scale.

This raises the question of how Jalysus acquires Burkholderia. The bean bug, Riptortus pedestris, acquires Burkholderia directly from the soil (Kikuchi et al., 2007). Fine-scale variation in the Burkholderia strains associated with Jalysus may therefore result from highly localized changes in strain abundances in the environment, for example from soil underneath the plant, and/or dust with bacterial cells blowing onto the plant. Another local source could include the tissue of the host plant itself. It is possible that plants could become infected with a particular Burkholderia strain (or strains); insects that colonize a plant could then be more likely to acquire the host plant's strain (Kaltenpoth and Flórez, 2020).

Alternatively, nymphs might acquire the symbiont vertically (from their parents) or horizontally from conspecific adults, at least some of the time. In our rearing experiments, 2 out of 26 nymphs acquired Burkholderia without intentional exposure to a symbiont source. It is possible that they acquired Burkholderia by vertical transmission via the egg surface, as occurs in symbioses between Burkholderia and blissid bugs (Boucias et al., 2012; Itoh et al., 2014) and perhaps also largid bugs (Takeshita et al., 2015). However, in our early work with J. wickhami we performed diagnostic PCR on a small number of eggs and never detected Burkholderia. Furthermore, the two unexpectedly Burkholderia-positive nymphs were reared together in the same box for their first four instars. They may therefore have acquired Burkholderia via contamination of the rearing box rather than vertical transmission.

Although evidence for occasional vertical transmission via the egg is equivocal, we found that horizontal bug-to-bug transmission can occur in J. wickhami, at least in the lab. In our rearing experiments, roughly one third of nymphs acquired Burkholderia when directly exposed to a single adult male and over $80 \%$ of nymphs acquired Burkholderia when reared on leaves that had previously hosted six adult insects. This demonstrates that adult-to-nymph transmission is not only possible, but likely at high insect densities and in the absence of another symbiont source. This is surprising given that bug-to-bug transmission was never observed in the bean 
bug, R. pedestris (Alydidae) despite continuous exposure of 107 nymphs to adults (Kikuchi et al., 2007) and suggests that the ability to transmit Burkholderia varies among the hemipteran families.

Jalysus' transmission capacity might be sex-specific. In Iteration 1 we used adult males as the Burkholderia source, whereas in Iteration 3 we used groups of six "source" adults that included males and females. Furthermore, source females were allowed to lay eggs (which were removed prior to introduction of the experimental nymphs). While the high Burkholderia colonization rate in Iteration 3 was likely due to the higher density of source adults, it is also possible that adult females transmit Burkholderia more successfully that adult males, and they might transmit it most actively while they are laying eggs. While highly speculative, either or both phenomena could be evolutionarily favorable given the demonstrated benefits Burkholderia provides to J. wickhami.

Overall, our results indicate that in the lab, (1) direct vertical transmission of Burkholderia is uncommon, if it occurs at all; (2) adult J. wickhami are capable of horizontal symbiont transmission; (3) the probability of transmission increases with the density of Burkholderia-colonized adults; and (4) transmission can occur without direct contact between nymphs and adults. This suggests that horizontal transmission of Burkholderia can occur via enrichment of the symbiont in the local environment by adult insects. While this possibility has been proposed previously (Itoh et al., 2018) to our knowledge this is the first empirical evidence that it can occur in a bug-Burkholderia symbiosis. It seems plausible that horizontal transmission via environmental enrichment also happens in the wild, given the insects' tendency to aggregate. Although Jalysus are not social, they often feed together in groups, sometimes clustering in multi-generation aggregations on flower stalks (Figure 1). Older individuals colonized by Burkholderia could therefore indirectly transmit the symbiont to nymphs by several mechanisms. They could inoculate water droplets on the plant, the leaf surface, or the internal tissues of the plant via feeding or feces (although related insects do not excrete live Burkholderia in their feces; Kikuchi et al., 2007) or they could inoculate the soil underneath the plant when they die, fall to the ground and decompose (though this mechanism was less likely in our lab since we removed dead insects from the cages). Occasional adult-to-nymph transmission could contribute to several of the patterns we observed, including the high similarity in strains hosted by insects collected at the same location and the prevalence of SV1 across most insect populations. Further research on the relative importance of these modes of Burkholderia acquisition will help elucidate the ecological maintenance and potential evolutionary origin of bugBurkholderia symbioses.

Finally, many of the insects in our study appeared to host two, three, or even more strains of Burkholderia. The fact that we extracted DNA from whole insect bodies might contribute to this pattern: although Burkholderia are usually housed in a specific location in the gut (crypts in the M4 region), some of the sequence variants observed in this study could have been located elsewhere in the insects' bodies. In a few cases, the appearance of multiple strains could be an artifact of the DADA2 algorithm. While powerful, any algorithm that differentiates at the level of a single base pair may occasionally mistake a strain with a single nucleotide polymorphism for two different strains. If this were the case, we would expect to see a consistent pair of strains present at the same relative abundances across insects. We do see this pattern with SV1 and SV21 (Figure 3). This pattern of cooccurrence is intriguing but therefore must be treated cautiously and requires further experimental investigation. However, many of the strains observed in multiply-infected insects did not display consistent pairings and frequencies, suggesting in these instances at least, multiple strains were indeed present. Multiple Burkholderia symbiont strains have been cultured from single insects in other bug-Burkholderia symbioses (Kikuchi et al., 2011) and, in general, multiple strain infection is more frequent in symbioses that involve horizontal or environmental acquisition (Ebert, 2013). Within the M4 different strains might occupy different crypts or compartments, facilitating coexistence. The presence of multiple symbiont strains within some individuals raises additional questions about the factors that determine which strain (or strains) an insect will host. Burkholderia strains belonging to the SBE (stinkbug-associated beneficial and environmental) clade have been shown to outcompete other clades of Burkholderia when colonizing the stinkbug gut (Itoh et al., 2019). Do SBE-clade Burkholderia strains also compete with one another? Is multiple-strain colonization and, potentially, competition - helpful or harmful to the host? Can strains displace each other? Do priority effects affect which strain colonizes an insect? These are fruitful avenues for future research.

We cannot yet say what associating with a variety of Burkholderia strains means functionally for the host. Jalysus may associate with many strains of Burkholderia because all strains are functionally equivalent to the insect. If this is the case, abundances of strains in an insect population might reflect abundances of SBE-clade Burkholderia in their local environment. However, strains likely vary in their competitive ability to colonize the host, as has been previously shown in $R$. pedestris (Itoh et al., 2019) which could skew strain abundances relative to the environmental pool even if strains are functionally equivalent from the insect's perspective. Alternatively or additionally, the benefits provided by different strains may be context-dependent. Perhaps strains that are highly abundant in western populations are more beneficial in western climates, eastern strains are, similarly, particularly beneficial in eastern climates, while SV1 (the widespread strain) is moderately beneficial across all climates. It is uncertain whether Jalysus can actively select strains matched to local conditions, however, a passive process could still result in local adaptation. For example, if insects acquire strains based on their frequency in the environment but some strains are more beneficial, the individuals that happen to host the "better" strains are more likely to survive and will therefore dominate the insect population. Furthermore, our rearing experiments suggest that adult Jalysus can transmit the symbiont to nymphs via enrichment of the local environment, and therefore may provide a baseline reservoir of Burkholderia for nymphs in their immediate vicinity. 


\section{DATA AVAILABILITY STATEMENT}

Raw Illumina sequences are available on the NCBI Sequence Read Archive under BioProject number PRJNA613319. All other data (Jalysus rearing data, Illumina SV table, SV taxonomic assignments, representative sequences, and sample metadata) are available as Supplementary Material.

\section{AUTHOR CONTRIBUTIONS}

$\mathrm{AR}$ and $\mathrm{MH}$ designed the study. AR and MT collected the samples. AR performed the experiment, processed the samples, analyzed the data, and wrote the first draft of the manuscript. All authors contributed to manuscript revision and read and approved the submitted version.

\section{FUNDING}

This work was supported by an NIH PERT postdoctoral fellowship through the Center for Insect Science to AR (grant

\section{REFERENCES}

Anbutsu, H., Moriyama, M., Nikoh, N., Hosokawa, T., Futahashi, R., Tanahashi, M., et al. (2017). Small genome symbiont underlies cuticle hardness in beetles. Proc. Natl. Acad. Sci. U.S.A. 114, E8382-E8391. doi: 10.1073/pnas.1712857114

Anders, S., and Huber, W. (2010). Differential expression analysis for sequence count data via mixtures of negative binomials. Nat. Preced. 11:R106. doi: 10. 1038/npre.2010.4282.2

Boucias, D. G., Garcia-Maruniak, A., and Cherry, R. (2012). Detection and characterization of bacterial symbionts in the heteropteran. Blissus insularis. FEMS Microbiol. Ecol. 82, 629-641. doi: 10.1111/j.1574-6941.2012.01433.x

Buchner, P. (1965). Endosymbiosis of Animals With Plant Microorganisms. New York, NY: Interscience Publishers.

Callahan, B. J., McMurdie, P. J., Rosen, M. J., and Uros, G. (2016). DADA2: highresolution sample inference from Illumina amplicon data. Nat. Methods 13, 581-583. doi: 10.1038/nmeth.3869

Cohen, A. C. (1985). Simple method for rearing the insect predator geocoris punctipes (Heteroptera: Lygaeldae) on a meat diet. J. Econ. Entomol. 78, 11731175.

Costello, M., Fleharty, M., and Abreu, J. (2018). Characterization and remediation of sample index swaps by non-redundant dual indexing on massively parallel sequencing platforms. BMC Genomics 19:332. doi: 10.1186/s12864-018-4703-0

Davis, N. M., Proctor, D. I. M., Holmes, S. P., and Watson, R. (2018). Simple statistical identification and removal of contaminant sequences in marker-gene and metagenomics data. Microbiome 6, 1-14. doi: 10.1186/s40168-018-0605-2

Douglas, A. E. (1998). Host benefit and the evolution of specialization in symbiosis. Heredity 81, 599-603.

Douglas, A. E. (2011). Lessons from studying insect symbioses. Cell Host Microbe 10, 359-367. doi: 10.1016/j.chom.2011.09.001

Douglas, A. E. (2015). Multiorganismal insects: diversity and function of resident microorganisms. Annu. Rev. Entomol. 60, 17-34. doi: 10.1146/annurev-ento010814-020822

Ebert, D. (2013). The epidemiology and evolution of symbionts with mixed-mode transmission. Annu. Rev. Ecol. Evol. Syst. 44, 623-643. doi: 10.1146/annurevecolsys-032513-100555

Elsey, K. D., and Stinner, R. E. (1971). Biology of Jalysus spinosus, an insect predator found on tobacco. Ann. Entomol. Soc. Am. 64, 779-783.

Garcia, J. R., Laughton, A. M., and Malik, Z. (2014). Partner associations across sympatric broad-headed bug species and their environmentally acquired bacterial symbionts. Mol. Ecol. 23, 1333-1347. doi: 10.1111/mec.12655 number K12GM000708) and by a United States Department of Agriculture NIFA grant (2019-67013-29407) to MH, AR, and David Baltrus.

\section{ACKNOWLEDGMENTS}

We sincerely thank Suzanne Kelly, Shaira Whitaker, Catherine Gavin, Dana Johnson, and Ryan Comella for laboratory assistance, Al Wheeler, Billy Krimmel, and Pete Nelson for their advice and help in the field, and Wendy Moore for assistance building the phylogeny.

\section{SUPPLEMENTARY MATERIAL}

The Supplementary Material for this article can be found online at: https://www.frontiersin.org/articles/10.3389/fmicb. 2020.01276/full\#supplementary-material

Hamady, M., Walker, J. J., and Harris, J. K. (2008). Error-correcting barcoded primers for pyrosequencing hundreds of samples in multiplex. Nat. Methods 5, 235-237. doi: 10.1038/NMETH.1184

Hosokawa, T., Hironaka, M., and Inadomi, K. (2013). Diverse strategies for vertical symbiont transmission among subsocial stinkbugs. PLoS One 8:e65081. doi: 10.1371/journal.pone.0065081

Hosokawa, T., Kikuchi, Y., and Nikoh, N. (2006). Strict host-symbiont cospeciation and reductive genome evolution in insect gut bacteria. PLoS Biol. 4:e337. doi: 10.1371/journal.pbio.0040337

Illumina Inc. (2013). 16S Metagenomic Sequencing Library Preparation. Available online at: https://support.illumina.com/downloads/16s_metagenomic_ sequencing_library_preparation.html (accessed May 23, 2017).

Itoh, H., Aita, M., and Nagayama, A. (2014). Evidence of environmental and vertical transmission of burkholderia symbionts in the oriental chinch bug. Cavelerius saccharivorus (Heteroptera: Blissidae). Appl. Environ. Microbiol. 80, 5974-5983. doi: 10.1128/AEM.01087-14

Itoh, H., Hori, T., and Sato, Y. (2018). Infection dynamics of insecticide-degrading symbionts from soil to insects in response to insecticide spraying. ISME J. 12, 909-920. doi: 10.1038/s41396-017-0021-9

Itoh, H., Jang, S., and Takeshita, K. (2019). Host-symbiont specificity determined by microbe-microbe competition in an insect gut. Proc. Natl. Acad. Sci. U.S.A. 116, 22673-22682. doi: 10.1073/pnas.1912397116

Janson, E. M., Stireman, J. O., Singer, M. S., and Abbot, P. (2008). Phytophagous insect-microbe mutualisms and adaptive evolutionary diversification. Evolution 62, 997-1012. doi: 10.1111/j.1558-5646.2008.00348.x

Kaltenpoth, M., and Flórez, L. V. (2020). Versatile and dynamic symbioses between insects and burkholderia bacteria. Annu. Rev. Entomol. 65, 15-40. doi: 10.1146/ annurev-ento-011019-025025

Katoh, K., and Standley, D. M. (2013). MAFFT multiple sequence alignment software version 7: improvements in performance and usability. Mol. Biol. Evol. 30, 772-780. doi: 10.1093/molbev/mst010

Kikuchi, Y., Hayatsu, M., and Hosokawa, T. (2012a). Symbiont-mediated insecticide resistance. Proc Natl Acad Sci. U.S.A. 109:861. doi: 10.1073/pnas. 1200231109

Kikuchi, Y., Hosokawa, T., Nikoh, N., and Fukatsu, T. (2012b). Gut symbiotic bacteria in the cabbage bugs eurydema rugosa and eurydema dominulus (Heteroptera: Pentatomidae). Appl. Entomol. Zool. 47, 1-8. doi: 10.1007/s13355011-0081-7

Kikuchi, Y., Hosokawa, T., and Fukatsu, T. (2007). Insect-microbe mutualism without vertical transmission: a stinkbug acquires a beneficial gut symbiont 
from the environment every generation. Appl. Environ. Microbiol. 73, 43084316. doi: 10.1128/AEM.00067-07

Kikuchi, Y., Hosokawa, T., and Fukatsu, T. (2011). An ancient but promiscuous host-symbiont association between burkholderia gut symbionts and their heteropteran hosts. ISME J. 5, 446-460. doi: 10.1038/ismej.2010.150

Kikuchi, Y., Meng, X. Y. X., and Fukatsu, T. (2005). Gut symbiotic bacteria of the genus? Burkholderia in the broad-headed bugs Riptortus clavatus and Leptocorisa chinensis (Heteroptera: Alydidae). Appl. Environ. Microbiol. 71, 4035-4043. doi: 10.1128/AEM.71.7.4035

Kikuchi, Y., Ohbayashi, T., Jang, S., and Mergaert, P. (2020). Burkholderia insecticola triggers midgut closure in the bean bug Riptortus pedestris to prevent secondary bacterial infections of midgut crypts. ISME J. doi: 10.1038/s41396020-0633-3

Kikuchi, Y., Tada, A., and Musolin, D. L. (2016). Collapse of insect gut symbiosis under simulated climate change. MBio 7:e1578-16. doi: 10.1128/mBio. 01578-16

Klindworth, A., Pruesse, E., and Schweer, T. (2013). Evaluation of general $16 \mathrm{~S}$ ribosomal RNA gene PCR primers for classical and next-generation sequencing-based diversity studies. Nucleic Acids Res. 41, 1-11. doi: 10.1093/ nar/gks808

Knight, R., Vrbanac, A., Taylor, B. C., Aksenov, A., Callewaert, C., Debelius, J., et al. (2018). Best practices for analysing microbiomes. Nat. Rev. Microbiol. 16, 410-422. doi: 10.1038/s41579-018-0029-9

Kuechler, S. M., Matsuura, Y., Dettner, K., and Kikuchi, Y. (2016). Phylogenetically diverse burkholderia associated with midgut crypts of spurge bugs, Dicranocephalus spp. (Heteroptera: Stenocephalidae).Microbes Environ. 31, 145-153. doi: 10.1264/jsme2.ME16042

Martin, F. M., Uros, S., and Barker, D. G. (2017). Ancestral alliances: plant mutualistic symbioses with fungi and bacteria. Science 356:4501. doi: 10.1126/ science.aad 4501

Martin, M. (2011). Cutadapt removes adapter sequences from high-throughput sequencing reads. EMBnet J. 17, 1-10. doi: 10.14806/ej.17.1.200

McFall-Ngai, M., Hadfield, M. G., and Bosch, T. C. G. (2013). Animals in a bacterial world, a new imperative for the life sciences. Proc. Natl. Acad. Sci. U.S.A. 110, 3229-3236. doi: 10.1073/pnas.1218525110

McMurdie, P. J., and Holmes, S. (2013). Phyloseq: an R package for reproducible interactive analysis and graphics of microbiome census data. PLoS One 8:e61217. doi: 10.1371/journal.pone.0061217

McMurdie, P. J., and Holmes, S. (2014). Waste not, want not: why rarefying microbiome data is inadmissible. PLoS Comput. Biol. 10:e1003531. doi: 10.1371/ journal.pcbi.1003531

Miller, M. A., Pfeiffer, W., and Schwartz, T. (2010). "Creating the CIPRES science gateway for inference of large phylogenetic trees," in Proceedings of the Gateway Computing Environments Workshop (GCE), New Orleans, LA: IEEE, 1-8.

Moran, N. A., McCutcheon, J. P., and Nakabachi, A. (2008). Genomics and evolution of heritable bacterial symbionts. Annu. Rev. Genet. 42, 165-190. doi: 10.1146/annurev.genet.41.110306.130119
Ohbayashi, T., Futahashi, R., and Terashima, M. (2019). Comparative cytology, physiology and transcriptomics of burkholderia insecticola in symbiosis with the bean bug Riptortus pedestris and in culture. ISME J. 13, 1469-1483. doi: 10.1038/s41396-019-0361-8

Ohbayashi, T., Takeshita, K., Kitagawa, W., Nikoh, N., Koga, R., Meng, X.-Y., et al. (2015). Insect's intestinal organ for symbiont sorting. Proc. Natl. Acad. Sci. U.S.A. 112, E5179-E5188. doi: 10.1073/pnas.1511454112

Oksanen, J., Blanchet, F. G., and Kindt, R. (2015). Vegan: Community Ecology Package. R Package Version 2.3-0.

Quast, C., Pruesse, E., and Yilmaz, P. (2013). The SILVA ribosomal RNA gene database project: improved data processing and web-based tools. Nucleic Acids Res. 41, 590-596. doi: 10.1093/nar/gks1219

Rohland, N., and Reich, D. (2012). Cost-effective, high-throughput DNA sequencing libraries for multiplexed target capture. Genome Res. 22, 939-946. doi: $10.1101 /$ gr.128124.111.22

Salem, H., Florez, L., Gerardo, N., and Kaltenpoth, M. (2015). An out-of-body experience: the extracellular dimension for the transmission of mutualistic bacteria in insects. Proc. R. Soc. B Biol. Sci. 282:20142957. doi: 10.1098/rspb. 2014.2957

Stamatakis, A. (2014). RAxML version 8: a tool for phylogenetic analysis and post-analysis of large phylogenies. Bioinformatics 30, 1312-1313. doi: 10.1093/ bioinformatics/btu033

Takeshita, K., and Kikuchi, Y. (2017). Riptortus pedestris and Burkholderia symbiont: an ideal model system for insect-microbe symbiotic associations. Res. Microbiol. 168, 175-187.

Takeshita, K., Matsuura, Y., Itoh, H., Navarro, R., Hori, T., Sone, T., et al. (2015). Burkholderia of plant-beneficial group are symbiotically associated with bordered plant bugs (Heteroptera: Pyrrhocoroidea: Largidae). Microbes Environ. 30, 321-329. doi: 10.1264/jsme2.ME15153

Wang, Q., Garrity, G. M., Tiedje, J. M., and Cole, J. R. (2007). Naïve bayesian classifier for rapid assignment of rRNA sequences into the new bacterial taxonomy. Appl. Environ. Microbiol. 73, 5261-5267. doi: 10.1128/AEM.00 062-07

White, J. A. (2011). Caught in the act: rapid, symbiont-driven evolution. BioEssays 33, 823-829. doi: 10.1002/bies.201100095

Conflict of Interest: The authors declare that the research was conducted in the absence of any commercial or financial relationships that could be construed as a potential conflict of interest.

Copyright (c) 2020 Ravenscraft, Thairu, Hansen and Hunter. This is an open-access article distributed under the terms of the Creative Commons Attribution License (CC BY). The use, distribution or reproduction in other forums is permitted, provided the original author(s) and the copyright owner(s) are credited and that the original publication in this journal is cited, in accordance with accepted academic practice. No use, distribution or reproduction is permitted which does not comply with these terms. 